\title{
Stage II Intrahepatic Cholangiocarcinoma AJCC v7
}

National Cancer Institute

\section{Source}

National Cancer Institute. Stage II Intrahepatic Cholangiocarcinoma A/CC v7. NCI

Thesaurus. Code C88051.

Stage II includes: T2, N0, M0. T2: Solitary tumor with vascular invasion or multiple tumors, with or without vascular invasion. N0: No regional lymph node metastasis. M0: No distant metastasis. (from AJCC 7th Ed.) 